\title{
FAST TIME IMPLICIT-EXPLICIT DISCONTINUOUS GALERKIN METHOD FOR CONVECTION DOMINATED FLOW PROBLEMS*
}

\author{
FLORENT RENAC ${ }^{\dagger}$, CLAUDE MARMIGNON ${ }^{\ddagger}$, AND FRÉDÉRIC COQUEL ${ }^{\S}$
}

\begin{abstract}
An efficient and robust time integration procedure for a high-order discontinuous Galerkin method is introduced for solving unsteady second-order partial differential equations. The time discretization is based on an explicit formulation for the hyperbolic term and an implicit formulation for the parabolic term. The implicit procedure uses a fast iterative algorithm with reduced evaluation cost introduced in [Renac, Marmignon, and Coquel, SIAM J. Sci. Comput., 34, A370A394, 2012]. The method is here extended to convection dominated flow problems. A second-order discretization in time is achieved by decomposing the integrations of convective and diffusive terms with a splitting method. Numerical examples are presented for the linear convection-diffusion equation in one and two space dimensions. The performance of the present method is seen to be improved in terms of CPU time when compared to a full implicit discretization of the parabolic terms in a wide range of Peclet numbers.
\end{abstract}

Key words. Discontinuous Galerkin method, linear convection-diffusion equation, convection dominated problems, implicit-explicit time discretization, splitting method.

AMS subject classifications. $65 \mathrm{~N} 30,65 \mathrm{~N} 12$.

\section{Introduction}

Discontinuous Galerkin (DG) methods are high-order finite element discretizations and were introduced for the numerical simulation of the first-order hyperbolic neutron transport equation $[10,11]$. The method was later extended to nonlinear convection dominated flow problems with the use of a Runge-Kutta method for the time integration $[5,7]$. In recent years, there has been a strong interest for these techniques in the field of computational fluid dynamics, which has led to the introduction of discretization schemes for parabolic and elliptic equations. For more details, the reader is referred to the analysis of existing discretizations for pure elliptic problems [2], to the overview of recent progress in DG methods for compressible flows [9], and to references cited therein. The success of these methods lies in their high-order of accuracy and flexibility thanks to their high degree of locality. These properties make the DG method well suited to algorithm parallelization, $h p$-refinement, unstructured meshes, etc.

However, the association of a DG method to an explicit time discretization leads to a strong restriction on the time step due to the so-called Courant-Friedrichs-Levy (CFL) condition for stability of the numerical scheme [1,4]. Numerical experiments on the resolution of the linear scalar convection equation have shown that for a $\operatorname{DG}(p)$ discretization with polynomials of degree $p$ and a $p+1$ Runge-Kutta method of order $p+1$, the CFL condition behaves as $\sigma \leq 1 /(2 p+1)$, where $\sigma=c \Delta t / h$ denotes the Courant number with $c>0$ the convection velocity, $\Delta t$ the time step, and $h$ the element size [7]. This property has been analytically demonstrated for a DG(1) discretization in [6] when using total variation diminishing Runge-Kutta methods from

\footnotetext{
*Received: November 3, 2011; accepted (in revised form): February 18, 2012. Communicated by Shi Jin.

†ONERA The French Aerospace Lab, 92320 Châtillon Cedex, France (florent.renac@onera.fr).

${ }^{\ddagger}$ ONERA The French Aerospace Lab, 92320 Châtillon Cedex, France (claude.marmignon@onera.fr).

$\S_{\text {CNRS }}$ and CMAP, Ecole Polytechnique, 91128 Palaiseau Cedex, France (frederic.coquel@cmap.polytechnique.fr).
} 
[13]. Moreover, the use of a forward Euler method leads to a prohibitive condition for stability: $\sigma \leq \mathcal{O}\left(h^{1 / 2}\right)[4]$.

The discretization of the parabolic terms requires an additional stability constraint on the von Neumann number $\sigma / R e_{h}$, which becomes more restrictive than the CFL condition for flow regions with small Peclet number $R e_{h}=c h / \nu$, with $\nu>0$ the diffusion coefficient. In high Reynolds number flows, the large variations in element size required to resolve all the spatial scales make the use of explicit integration techniques prohibitive. On the other hand, the application of fully implicit methods to a system of conservation laws leads to an algebraic system to be solved at each time step. The size of this system is equal to the total number of degrees of freedom (DOFs) of the discrete problem and may become very large for practical applications, such as turbulent flow simulations.

A robust and fast time integration procedure for a semi-implicit formulation of a DG discretization of convection-diffusion equations has been introduced in [12]. The method uses an implicit backward Euler scheme for the viscous terms, while convective terms are treated explicitly. A simplified implicit problem, based on a reduced communication between elements which share a common interface, is used for the resolution of the discrete problem at each time step. This assumption follows from the natural hierarchy of the basis of the discrete function space: the communication between elements is assumed to be dominated by the coupling between DOFs associated to the components of the basis of lowest-order. After solving an implicit problem of reduced size for the low-order DOFs only, a local reconstruction, based on the stencil of the DG discretization, is used for evaluating higher-order DOFs. Numerical experiments on one-dimensional (1D) and two-dimensional (2D) nonlinear convection-diffusion equations have highlighted better performances of the present method in terms of CPU time when compared to a full implicit discretization of the viscous terms. This simplification also keeps the locality, accuracy, and stability properties of the original method. A von Neumann stability analysis carried out in [12] for a linear scalar convection-diffusion equation indicated that necessary conditions for stability of the full and simplified methods for $p=1$ read

$$
\sigma R e_{h} \leq 2, \quad R e_{h} \leq 6 .
$$

The first condition corresponds to a restriction on the time step of the form $\Delta t \leq 2 \nu / c^{2}$, while the second condition makes the implicit-explicit time integration inappropriate for convection dominated flow problems. In the present study, we propose to modify the method introduced in [12] and adapt it to unsteady convection dominated flows in order to remove the stability constraints (1.1). High-order accuracy in time is achieved through a second-order fast explicit-implicit decomposition method based on a Strang splitting [14]. The hyperbolic problem is treated explicitly, while the simplified implicit discretization from [12] is used for the parabolic problem.

\section{Model problem and discretization}

The discussion in this paper focuses on the discretization of linear scalar convection-diffusion equations with a DG method. Let $\Omega \subset \mathbb{R}^{d}$ be a bounded domain, where $d$ is the space dimension, and consider the problem

$$
\partial_{t} u+\nabla \cdot(\mathbf{c}(\mathbf{x}) u)-\nabla \cdot(\mathbf{B}(\mathbf{x}) \nabla u)=s(\mathbf{x}, t) \quad \text { in } \Omega,
$$

which describes the evolution of the scalar quantity $u(\mathbf{x}, t)$ convected in a velocity field $\mathbf{c}(\mathbf{x}) \in \mathbb{R}^{d}$ and subject to diffusion modelled by the diffusivity tensor $\mathbf{B}(\mathbf{x}) \in$ 
$\mathbb{R}^{d \times d}$ which is assumed to be symmetric and positive definite. The forcing function $s(\mathbf{x}, t) \in L^{2}(\Omega)$ represents production or destruction of the quantity $u$. The problem is supplemented with the initial condition $u(\cdot, 0)=u_{0}(\cdot)$ in $\Omega$, as well as Dirichlet and Neumann boundary conditions $u=u_{D}$ on $\Gamma_{D}$ and $\mathbf{B} \nabla u \cdot \mathbf{n}=g_{N}$ on $\Gamma_{N}$ with $\partial \Omega=$ $\Gamma_{D} \cup \Gamma_{N}$. We assume that $u_{D} \in L^{2}\left(\Gamma_{D}\right)$ and $g_{N} \in L^{2}\left(\Gamma_{N}\right)$. By $\mathbf{n}$ we denote the unit outward normal vector to $\partial \Omega$.

2.1. Discontinuous Galerkin formulation. The aim of the present work is to derive a fast time marching method for the numerical resolution of (2.1) with a DG space discretization. The domain $\Omega$ is partitioned into a shape-regular mesh $\Omega_{h}$ consisting of $N$ nonoverlapping and nonempty simplicies $\kappa$ of characteristic size $h:=\min \left\{\operatorname{diam}(\kappa), \kappa \in \Omega_{h}\right\}$, where $\operatorname{diam}(\kappa)$ is the diameter of the largest ball included in $\kappa$. We define the set of interior faces $\mathcal{E}_{i}$ and boundary faces $\mathcal{E}_{b}$ and further define $\mathcal{E}_{h}=\mathcal{E}_{i} \cup \mathcal{E}_{b}$, the union of all open $(d-1)$-dimensional faces in $\Omega_{h}$. The set of boundary faces may be divided into $\mathcal{E}_{b}=\mathcal{E}_{D} \cup \mathcal{E}_{N}$ consisting of faces of $\Gamma_{D}$ and $\Gamma_{N}$, respectively.

We look for approximate solutions in the function space of discontinuous polynomials $\mathcal{V}_{h}^{p}=\left\{\varphi \in L^{2}\left(\Omega_{h}\right):\left.\varphi\right|_{\kappa} \circ F_{\kappa} \in \mathcal{P}_{p}\left(I^{d}\right), \kappa \in \Omega_{h}\right\}$, where $\mathcal{P}_{p}\left(I^{d}\right)$ denotes the space of functions formed by tensor products of polynomials and total degree at most $p$ on the master element $I^{d}$, where $I=[-1,1]$. Each physical element $\kappa$ is the image of $I^{d}$ through the mapping $F_{\kappa}$. The numerical solution of problem (2.1) is sought under the form

$$
u_{h}(\mathbf{x}, t)=\sum_{l=1}^{N_{p}} \phi^{l}(\mathbf{x}) U_{\kappa}^{l}(t), \quad \forall \mathbf{x} \in \kappa, \quad \kappa \in \Omega_{h}, \quad \forall t \geq 0,
$$

where $U_{\kappa}^{l}$ are the DOFs in the element $\kappa$. The subset $\left(\phi^{1}, \ldots, \phi^{N_{p}}\right)$ constitutes a hierarchical and orthogonal modal basis of $\mathcal{V}_{h}^{p}$ restricted onto a given element and $N_{p}=\prod_{i=1}^{d}(p+i) / i$ is its dimension. In this work, we will use Legendre polynomials as basis in one dimension and the Dubiner basis [8] in two dimensions.

The second method of Bassi and coworkers uses lifting operators to account for discontinuity of the numerical solution across interfaces when evaluating its gradient [3]. The face-based formulation of this discretization for problem (2.1) reads: find $u_{h}$ in $\mathcal{V}_{h}^{p}$ such that for all $v_{h}$ in $\mathcal{V}_{h}^{p}$ we have

$$
\int_{\Omega_{h}} v_{h} \partial_{t} u_{h} d \mathbf{x}+\mathcal{L}_{c}\left(u_{h}, v_{h}\right)+\mathcal{L}_{v}\left(u_{h}, v_{h}\right)+\mathcal{L}_{s}\left(v_{h}\right)=0 .
$$

Hereafter, we will use the notations $\left\{v_{h}\right\}=\left(v_{h}^{+}+v_{h}^{-}\right) / 2$ and $\llbracket v_{h} \rrbracket=v_{h}^{+}-v_{h}^{-}$, which denote the average and jump operators defined for a given interface $e$. Here, $v_{h}^{+}$and $v_{h}^{-}$are the traces of $v_{h}$ on the interface $e$ taken from within the interior of the element $\kappa^{+}$and the interior of the neighbouring element $\kappa^{-}$, respectively (see figure 2.1).

The discretization of the hyperbolic term in (2.3) reads

$$
\begin{aligned}
\mathcal{L}_{c}\left(u_{h}, v_{h}\right)= & -\int_{\Omega_{h}} u_{h} \mathbf{c} \cdot \nabla_{h} v_{h} d \mathbf{x}+\int_{\mathcal{E}_{i}} \llbracket v_{h} \rrbracket h_{c}\left(u_{h}^{+}, u_{h}^{-}, \mathbf{n}\right) d s \\
& +\int_{\mathcal{E}_{N}} v_{h}^{+} u_{h}^{+} \mathbf{c} \cdot \mathbf{n} d s+\int_{\mathcal{E}_{D}} v_{h}^{+} u_{D} \mathbf{c} \cdot \mathbf{n} d s,
\end{aligned}
$$

where $\mathbf{n}$ denotes the unit outward normal vector to the element $\kappa^{+}$(see figure 2.1). We use a standard upwind discretization flux as numerical convective flux: $h_{c}\left(u_{h}^{+}, u_{h}^{-}, \mathbf{n}\right)=$ 


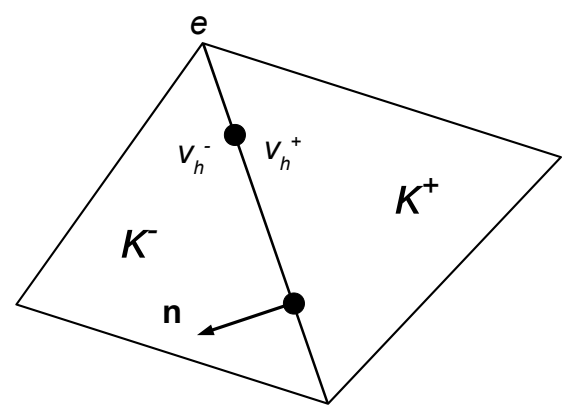

FIG. 2.1. Inner and exterior elements $\kappa^{+}$and $\kappa^{-}$and definitions of traces $v_{h}^{ \pm}$on the interface $e$ and of the unit outward normal vector $\mathbf{n}$.

$\left\{u_{h} \mathbf{c}\right\} \cdot \mathbf{n}+\frac{|\mathbf{c} \cdot \mathbf{n}|}{2} \llbracket u_{h} \rrbracket ;$ see for instance [7]. The bilinear and linear forms in (2.3) are defined by

$$
\begin{aligned}
\mathcal{L}_{v}\left(u_{h}, v_{h}\right)= & \int_{\Omega_{h}} \mathbf{B}\left(\nabla_{h} u_{h}+\mathbf{R}_{\mathbf{h}}\right) \cdot \nabla_{h} v_{h} d \mathbf{x}-\int_{\mathcal{E}_{i}} \llbracket v_{h} \rrbracket\left\{\mathbf{B}\left(\nabla_{h} u_{h}+\mathbf{r}_{\mathbf{h}}^{\mathbf{e}}\right)\right\} \cdot \mathbf{n} d s \\
& -\int_{\mathcal{E}_{D}} v_{h}^{+} \mathbf{B}\left(\nabla_{h} u_{h}^{+}+\mathbf{r}_{\mathbf{h}}^{\mathbf{e}}\right) \cdot \mathbf{n} d s-\int_{\mathcal{E}_{N}} v_{h}^{+} g_{N} d s,
\end{aligned}
$$

and

$$
\mathcal{L}_{s}\left(v_{h}\right)=-\int_{\Omega_{h}} v_{h} s(\mathbf{x}, t) d \mathbf{x} .
$$

The so-called global lifting operator $\mathbf{R}_{\mathbf{h}}$ is weakly defined as the sum of local lifting operators $\mathbf{r}_{\mathbf{h}}^{\mathbf{e}}$ that have support on elements adjacent to $e$ and are solutions of the following problem: for all $v_{h}$ in $\mathcal{V}_{h}^{p}$ we have $\int_{\kappa^{+} \cup \kappa^{-}} v_{h} \mathbf{r}_{\mathbf{h}}^{\mathbf{e}} d \mathbf{x}=-\int_{e}\left\{v_{h}\right\} \llbracket u_{h} \rrbracket \mathbf{n} d s$ on internal faces and $\int_{\kappa^{+}} v_{h} \mathbf{r}_{\mathbf{h}}^{\mathbf{e}} d \mathbf{x}=-\int_{e} v_{h}^{+}\left(u_{h}^{+}-u_{D}\right) \mathbf{n} d s$ on Dirichlet boundary faces. We refer to [3] for further details on this method.

2.2. Time discretization. The semi-discrete equation (2.3) is discretized in time by means of a second-order Strang splitting method [14]. Introducing a time step $\Delta t$ and defining $t^{(n)}=n \Delta t$, the solution is advanced in time in three substeps on the interval $\left(t^{(n)}, t^{(n+1)}\right]$. First, the hyperbolic problem is solved for $u_{h}^{c}$ on the time interval $\left(t^{(n)}, t^{(n)}+\frac{\Delta t}{2}\right]$ :

$$
\int_{\Omega_{h}} v_{h} \partial_{t} u_{h}^{c} d \mathbf{x}+\mathcal{L}_{c}\left(u_{h}^{c}, v_{h}\right)=0, \quad \forall t \in\left(t^{(n)}, t^{(n)}+\frac{\Delta t}{2}\right], \quad \forall v_{h} \in \mathcal{V}_{h}^{p},
$$

with initial condition $u_{h}^{c}\left(\cdot, t^{(n)}\right)=u_{h}\left(\cdot, t^{(n)}\right)$. Then, the parabolic problem with source term is solved for $u_{h}^{v}$ on the time interval $\left(t^{(n)}, t^{(n)}+\Delta t\right]$ :

$$
\int_{\Omega_{h}} v_{h} \partial_{t} u_{h}^{v} d \mathbf{x}+\mathcal{L}_{v}\left(u_{h}^{v}, v_{h}\right)+\mathcal{L}_{s}\left(v_{h}\right)=0, \quad \forall t \in\left(t^{(n)}, t^{(n)}+\Delta t\right], \quad \forall v_{h} \in \mathcal{V}_{h}^{p},
$$

with $u_{h}^{v}\left(\cdot, t^{(n)}\right)=u_{h}^{c}\left(\cdot, t^{(n)}+\frac{\Delta t}{2}\right)$. Finally, the hyperbolic problem is solved on the time interval $\left(t^{(n)}+\frac{\Delta t}{2}, t^{(n)}+\Delta t\right]$ :

$$
\int_{\Omega_{h}} v_{h} \partial_{t} u_{h}^{c} d \mathbf{x}+\mathcal{L}_{c}\left(u_{h}^{c}, v_{h}\right)=0, \quad \forall t \in\left(t^{(n)}+\frac{\Delta t}{2}, t^{(n)}+\Delta t\right], \quad \forall v_{h} \in \mathcal{V}_{h}^{p},
$$


with $u_{h}^{c}\left(\cdot, t^{(n)}+\frac{\Delta t}{2}\right)=u_{h}^{v}\left(\cdot, t^{(n)}+\Delta t\right)$. The solution of equation (2.3) at time $t^{(n+1)}$ is thus $u_{h}\left(\cdot, t^{(n+1)}\right)=u_{h}^{c}\left(\cdot, t^{(n)}+\Delta t\right)$. We use a Heun's method for the time discretization of hyperbolic problems (2.7) and (2.9). For example, for (2.7) we apply

$$
\begin{aligned}
\mathbf{U}_{c}^{\star} & =\mathbf{U}^{(n)}-\frac{\Delta t}{2} \mathbf{M}^{-1} \mathbf{L}_{\mathbf{c}}\left(\mathbf{U}^{(n)}\right), \\
\mathbf{U}_{c}^{\left(n+\frac{1}{2}\right)} & =\frac{1}{2}\left(\mathbf{U}_{c}^{\star}+\mathbf{U}^{(n)}-\frac{\Delta t}{2} \mathbf{M}^{-1} \mathbf{L}_{\mathbf{c}}\left(\mathbf{U}_{c}^{\star}\right)\right),
\end{aligned}
$$

with $\mathbf{U}$ the vector of DOFs, $\mathbf{L}_{\mathbf{c}}(\mathbf{U})$ the residual vector defined by the discrete form (2.4), and where $\mathbf{M}$ denotes the diagonal mass matrix. The method (2.10) is explicit and formally second-order accurate in time [13].

Problem (2.8) is discretized in time with an implicit second-order Crank-Nicolson method and reduces to the following linear system:

$$
\mathbf{A}\left(\mathbf{U}_{v}^{(n+1)}-\mathbf{U}_{c}^{\left(n+\frac{1}{2}\right)}\right)=-\mathbf{L}_{\mathbf{v}}\left(\mathbf{U}_{c}^{\left(n+\frac{1}{2}\right)}\right)-\mathbf{L}_{\mathbf{s}},
$$

with $\mathbf{L}_{\mathbf{v}}\left(\mathbf{U}_{c}^{\left(n+\frac{1}{2}\right)}\right)=\mathbf{L}_{\mathbf{v}} \mathbf{U}_{c}^{\left(n+\frac{1}{2}\right)}$ and $\mathbf{L}_{\mathbf{s}}$ the residual vectors defined by the discrete forms (2.5) and (2.6). The so-called implicit matrix is defined by $\mathbf{A}=\frac{1}{\Delta t} \mathbf{M}+\frac{1}{2} \mathbf{L}_{\mathbf{v}}$ and is an asymmetric real matrix of size $N \times N_{p}$ since $\mathbf{L}_{\mathbf{v}}$ is. We use a preconditioned biconjugate gradient method for solving the linear system (2.11) at each time step. The inversion cost of $\mathbf{A}$ may become prohibitive in practical applications where $N$ is large and where accuracy is looked for. To circumvent this shortcoming, we propose the use of the modification of the implicit problem (2.11) introduced in [12] for simplifying its resolution. The method consists in reducing the coupling between DOFs in adjacent elements, thereby allowing the resolution of an implicit problem of lower size. For that purpose, let $p_{s}$ be a user-defined integer that separates low-order DOFs associated to components of degree $0 \leq q \leq p_{s}$ in the functional basis and high-order DOFs $p_{s}<q \leq p$. The simplified method is a two-steps algorithm: first, an implicit problem is solved for the low-order DOFs only, then higher-order DOFs are locally reconstructed. This method simplifies the task of solving the algebraic system. The reader is referred to [12] for more details on the method. In the following, the discrete problem with the full implicit method (2.11) will be referred to as the FULL method, and the simplified implicit method from [12] will be referred to as the $\operatorname{SIMP} p_{s}$ method. We now compare the properties and performances of both methods in the next sections.

\section{3. von Neumann analysis}

A von Neumann analysis is here performed in order to derive practical stability constraints on the numerical scheme parameters. For the sake of simplicity, we consider the 1D linear convection-diffusion equation

$$
\partial_{t} u+c \partial_{x} u-\nu \partial_{x x} u=0, \quad \text { in }(0,1] \times(0,+\infty),
$$

with $\nu>0, c>0$, and a periodic boundary condition $u(0, \cdot)=u(1, \cdot)$. Equation (3.1) admits Fourier modes of the form $u(x, t)=e^{i(k x-\omega t)}$ as exact solutions, where $k$ is the prescribed real wavenumber and $\omega$ is the corresponding complex frequency satisfying the dispersion relation $\omega=k c-i \nu k^{2}$ with $i^{2}=-1$. A similar decomposition is performed for the numerical solution $u_{h}$. The domain is first divided into regular 


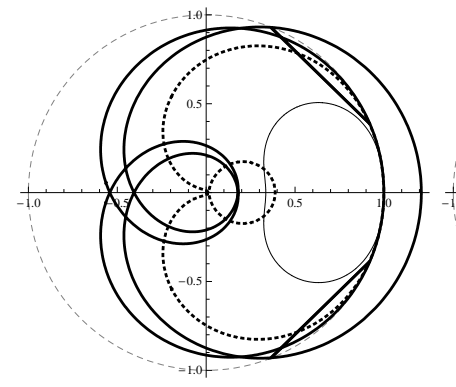

(a) $R e_{h}=1000$, FULL

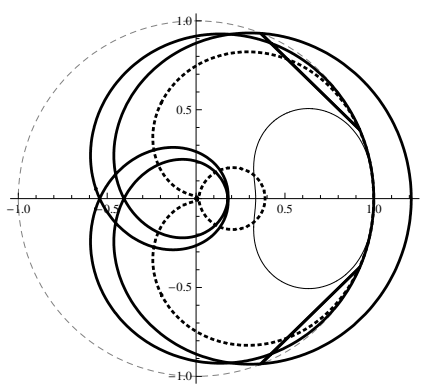

(b) $R e_{h}=1000$, SIMP0

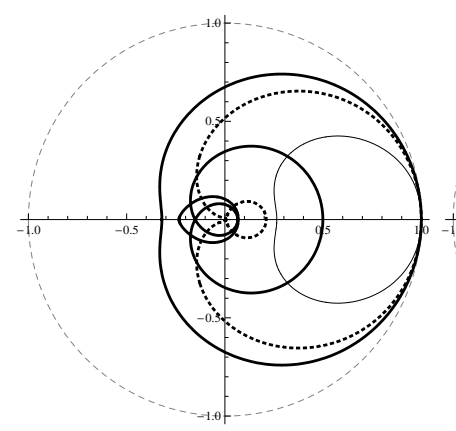

(c) $R e_{h}=10$, FULL

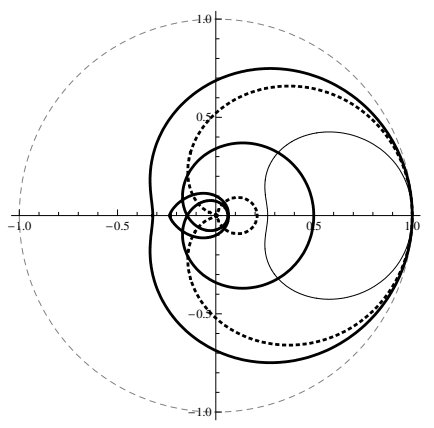

(d) $R e_{h}=10$, SIMP0

FIG. 3.1. Distributions of eigenvalues of the amplification matrix in the complex plane when $\theta$ varies within the range $(-\pi, \pi]$. Results are given for $p=1$ and $\sigma=0.2$ (thin lines), $\sigma=0.5$ (thick dotted lines), and $\sigma=0.7$ (thick lines). The unit circle $\mathcal{C}(0,1)$ in dashed gray denotes the stability domain.

elements of size $h$. Introducing the reduced wavenumber $\theta=k h$, the condition for stability that any error should remain uniformly bounded for $n \rightarrow \infty$ at fixed time step $\Delta t$ can be written as $\rho(\mathbf{G}) \leq 1$ for all $\theta$ in $(-\pi, \pi]$, where $\rho(\mathbf{G})$ denotes the spectral radius of the amplification matrix between times $t^{(n)}$ and $t^{(n+1)}$. Details on the derivation of the amplification matrices of both methods are given in Appendix A.

The eigenspectra of amplification matrices for numerical schemes of the FULL and SIMP0 methods are plotted in figures 3.1 and 3.2 for $p=1$ and $p=2$, respectively. Results are obtained for different values of the Courant and Peclet numbers. We note that the amplification matrix has multiple eigenvalues corresponding to multiple DOFs per element. Both methods present the same stability properties. Indeed, a stability analysis in the low wavenumber limit $\theta=0$ shows that a necessary condition for stability of both methods for $p=1$ reads

$$
\left|\frac{R e_{h}-6 \sigma}{R e_{h}+6 \sigma}\right|\left(1-3 \sigma+\frac{9 \sigma^{2}}{2}\right)^{2} \leq 1 .
$$

In the convective limit $R e_{h} \rightarrow \infty$, one recovers the condition $\sigma \leq 2 / 3$ for stability of the DG(1) discretization with a Heun's method (2.10) on half a time step [6]. Moreover, equation (3.2) shows that diffusion slightly enhances the stability of the numerical scheme in agreement with the analysis at low Peclet number in figures $3.1 \mathrm{c}, \mathrm{d}$ and $3.2 \mathrm{c}, \mathrm{d}$. Analytical stability conditions for $p=2$ are still an open problem but figure 


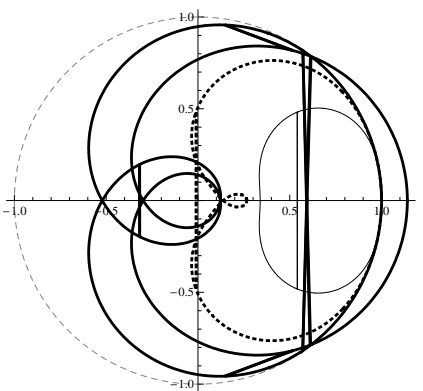

(a) $R e_{h}=1000$, FULL

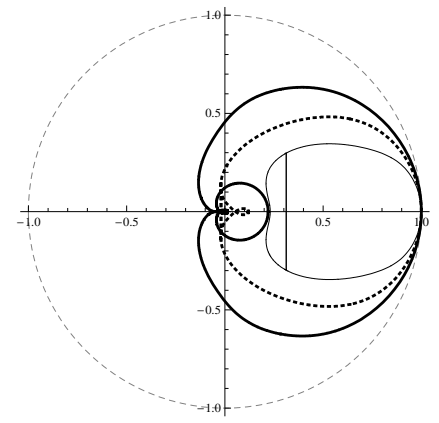

(c) $R e_{h}=10$, FULL

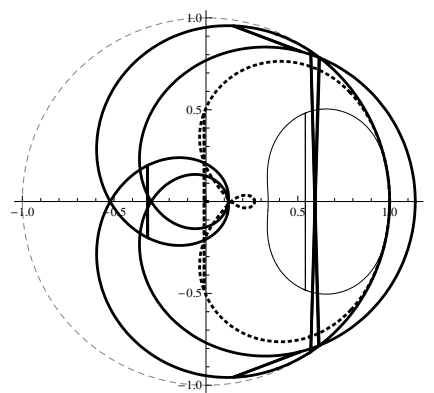

(b) $R e_{h}=1000$, SIMP0

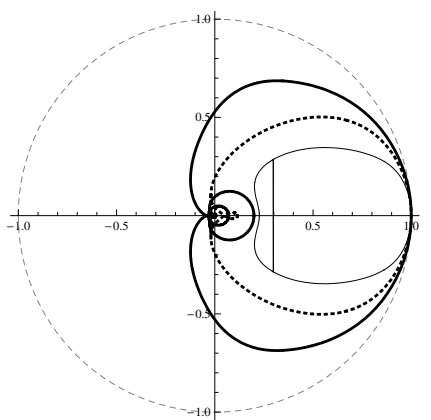

(d) $R e_{h}=10$, SIMP0

FIG. 3.2. Distributions of eigenvalues of the amplification matrix in the complex plane when $\theta$ varies within the range $(-\pi, \pi]$. Results are given for $p=2$ with $\sigma=0.1$ (thin lines), $\sigma=0.2$ (thick dotted lines), and $\sigma=0.35$ (thick lines). The unit circle $\mathcal{C}(0,1)$ in dashed gray denotes the stability domain.

3.2 clearly shows that the SIMP0 method does not alter the spectral properties of the numerical scheme in contrast to what was observed in the limit $R e_{h} \ll 1[12]$.

\section{Numerical experiments}

In this section we present numerical experiments in one and two dimensions to illustrate the performances of the simplification of the implicit time discretization.

4.1. One-dimensional unsteady convection-diffusion equation. In this example we let $\Omega=[0,1]$ and consider the following problem:

$$
\begin{aligned}
\partial_{t} u+c \partial_{x} u-\nu \partial_{x x} u & =0 & & \text { in }(0,1] \times(0, T], \\
u(x, 0) & =\sin (2 \pi x) & & \text { in }[0,1],
\end{aligned}
$$

with periodic boundary conditions, $c=1$, and $\nu>0$. The exact solution reads $u(x, t)=$ $\sin (2 \pi(x-c t)) e^{-4 \pi^{2} \nu t}$. The problem (4.1) is solved by using the method introduced in $\S 2$. The domain is discretized with an uniform grid $\Omega_{h}=\cup_{j=1}^{N}[(j-1) h, j h]$ with $h=1 / N$. We first investigate the asymptotic accuracy of the DG discretization under time step refinement. Table 4.1 presents the $L^{2}$ - and $L^{\infty}$-norms of the error $e_{h} \equiv u-u_{h}$ as a function of the nondimensional time step $\sigma$. To isolate the temporal convergence behaviour as much as possible, effects of spatial discretization errors are minimized by using a sufficiently refined mesh with $N=640$ elements and a high polynomial degree $p=3$. As expected, both schemes achieve optimal convergence $\left\|e_{h}\right\|_{2} \sim \sigma^{2}$ as $\sigma$ tends 
to zero. Moreover, error levels are identical between both methods indicating that the implicit simplification does not alter the error of the numerical scheme. This property was seen to hold for all experiments presented throughout this study so that the SIMP method may be used for the resolution of unsteady flow problems. The accuracy of the method under space refinement is investigated in table 4.2 for polynomial degrees $1 \leq p \leq 5$. Here again, we use Courant number values sufficiently small to isolate the space discretization error. These values lie in the range $10^{-5} \leq \sigma \leq 0.6$ for results shown in table 4.2. The $L^{2}$-norm of the error is seen to behave as $\left\|e_{h}\right\|_{2} \sim h^{p+1}$ in agreement with previous theoretical studies $[2,12]$ and results are again identical between both methods.

\begin{tabular}{|l|c|c|c|c|}
\hline & \multicolumn{2}{|c|}{ FULL method } & \multicolumn{2}{c|}{ SIMP0 method } \\
\hline$\sigma$ & $\left\|u-u_{h}\right\|_{2}$ & order & $\left\|u-u_{h}\right\|_{2}$ & order \\
\hline 0.2 & $1.78396 E-07$ & - & $1.78395 E-07$ & - \\
0.1 & $4.45991 E-08$ & 2.00 & $4.46027 E-08$ & 2.00 \\
0.05 & $1.11497 E-08$ & 2.00 & $1.11507 E-08$ & 2.00 \\
0.025 & $2.78740 E-09$ & 2.00 & $2.78765 E-09$ & 2.00 \\
0.0125 & $6.96821 E-10$ & 2.00 & $6.96888 E-10$ & 2.00 \\
\hline$\sigma$ & $\left\|u-u_{h}\right\|_{\infty}$ & order & $\left\|u-u_{h}\right\|_{\infty}$ & order \\
\hline 0.2 & $2.52290 E-07$ & - & $2.52348 E-07$ & - \\
0.1 & $6.30726 E-08$ & 2.00 & $6.30867 E-08$ & 2.00 \\
0.05 & $1.57681 E-08$ & 2.00 & $1.57737 E-08$ & 2.00 \\
0.025 & $3.94199 E-09$ & 2.00 & $3.94443 E-09$ & 2.00 \\
0.0125 & $9.85454 E-10$ & 2.00 & $9.86581 E-10$ & 2.00 \\
\hline
\end{tabular}

TABLE 4.1. $1 D$ problem: convergence of the FULL and SIMP methods under time step refinement and orders of accuracy. Results were obtained with $T=0.25, N=640, R_{h}=100, p=3$, and $p_{s}=0$.

In order to evaluate the relative efficiency of the FULL and SIMP methods, we define the speedup as the ratio of CPU time needed by the FULL method to CPU time with the SIMP method:

$$
\text { speedup }=\frac{\text { CPU time }(\text { FULL })}{\text { CPU time }(\text { SIMP })} .
$$

Results are given in table 4.3. The value of $p_{s}$ that separates low- and high-order DOFs is the lowest allowing to recover the same error levels as the FULL method. This choice ensures that a fair comparison between CPU time for all configurations can be made. We note a slight increase of the speedup values as $p$ is increased in agreement with previous experiments for low Peclet number flows $R_{h} \leq 1$ [12]. Speedups were nevertheless seen to reach larger values in the limit $R e_{h} \ll 1$ with the previous method. However, the present method allows CPU time savings on a wide range of Peclet numbers and speedups are almost independent of $R e_{h}$.

4.2. Two-dimensional unsteady convection-diffusion equation. In this second example, we consider the following problem with periodic boundary conditions in $\Omega=[0,1]^{2}$ :

$$
\begin{aligned}
\partial_{t} u+\nabla \cdot(\mathbf{c} u)-\nabla \cdot(\mathbf{B}(\mathbf{x}) \nabla u) & =s(\mathbf{x}, t) & & \text { in } \Omega \times(0, T], \\
u(\mathbf{x}, 0) & =\frac{1}{4} e^{-\frac{\left(\mathbf{x}-\mathbf{x}_{0}(0)\right)^{2}}{R^{2}}} & & \text { in } \Omega,
\end{aligned}
$$




\begin{tabular}{|c|c|c|c|c|c|}
\hline & & \multicolumn{2}{|c|}{ FULL method } & \multicolumn{2}{c|}{ SIMP0 method } \\
\hline$p$ & $h$ & $\left\|u-u_{h}\right\|_{2}$ & order & $\left\|u-u_{h}\right\|_{2}$ & order \\
\hline 1 & $1 / 5$ & $5.65759 E-01$ & - & $5.65759 E-01$ & - \\
1 & $1 / 10$ & $1.49354 E-01$ & 1.92 & $1.49354 E-01$ & 1.92 \\
1 & $1 / 20$ & $3.72136 E-02$ & 2.00 & $3.72136 E-02$ & 2.00 \\
1 & $1 / 40$ & $9.30274 E-03$ & 2.00 & $9.30274 E-03$ & 2.00 \\
\hline 2 & $1 / 5$ & $5.29312 E-02$ & - & $5.29312 E-02$ & - \\
2 & $1 / 10$ & $6.58149 E-03$ & 3.00 & $6.58149 E-03$ & 3.00 \\
2 & $1 / 20$ & $8.18270 E-04$ & 3.01 & $8.18270 E-04$ & 3.01 \\
2 & $1 / 40$ & $1.02174 E-04$ & 3.00 & $1.02174 E-04$ & 3.00 \\
\hline 3 & $1 / 5$ & $4.13523 E-03$ & - & $4.13523 E-03$ & - \\
3 & $1 / 10$ & $2.67339 E-04$ & 3.95 & $2.67339 E-04$ & 3.95 \\
3 & $1 / 20$ & $1.52136 E-05$ & 4.14 & $1.52136 E-05$ & 4.14 \\
3 & $1 / 40$ & $9.71220 E-07$ & 3.97 & $9.71220 E-07$ & 3.97 \\
\hline 4 & $1 / 5$ & $2.42659 E-04$ & - & $2.42659 E-04$ & - \\
4 & $1 / 10$ & $8.09728 E-06$ & 4.91 & $8.09728 E-06$ & 4.91 \\
4 & $1 / 20$ & $2.33398 E-07$ & 5.12 & $2.33398 E-07$ & 5.12 \\
4 & $1 / 40$ & $7.30361 E-09$ & 5.00 & $7.30361 E-09$ & 5.00 \\
\hline 5 & $1 / 5$ & $1.30595 E-05$ & - & $1.30595 E-05$ & - \\
5 & $1 / 10$ & $2.00299 E-07$ & 6.03 & $2.00299 E-07$ & 6.03 \\
5 & $1 / 20$ & $3.07250 E-09$ & 6.03 & $3.07250 E-09$ & 6.03 \\
5 & $1 / 40$ & $4.80344 E-11$ & 6.00 & $4.80344 E-11$ & 6.00 \\
\hline
\end{tabular}

TABLE 4.2. $1 D$ problem: convergence of the FULL and SIMP methods under mesh refinement and orders of accuracy. Results were obtained with $T=0.25, R e_{h}=100$, and $p_{s}=0$.

\begin{tabular}{|c|c|c|c|c|c|c|c|c|c|}
\hline & & \multicolumn{2}{|c|}{$R e_{h}=10$} & \multicolumn{2}{c|}{$R e_{h}=100$} & \multicolumn{2}{c|}{$R e_{h}=1000$} & \multicolumn{2}{c|}{$R e_{h}=10000$} \\
\hline$p$ & $\sigma$ & $p_{s}$ & speedup & $p_{s}$ & speedup & $p_{s}$ & speedup & $p_{s}$ & speedup \\
\hline 1 & 0.6 & 0 & 1.36 & 0 & 1.23 & 0 & 1.28 & 0 & 1.38 \\
2 & 0.3 & 0 & 1.36 & 0 & 1.34 & 0 & 1.40 & 0 & 1.36 \\
3 & 0.2 & 0 & 1.56 & 0 & 1.41 & 0 & 1.33 & 0 & 1.45 \\
4 & 0.1 & 1 & 1.47 & 0 & 1.42 & 0 & 1.30 & 0 & 1.37 \\
5 & 0.1 & 1 & 1.71 & 0 & 1.48 & 0 & 1.39 & 0 & 1.34 \\
\hline
\end{tabular}

TABLE 4.3. $1 D$ problem: speedups observed from experiments with $T=0.25$ and $N=640$.

with longitudinal advection $\mathbf{c}=(1,0)^{\top}$ and a diffusion tensor of the form $\mathbf{B}(\mathbf{x})=$ $\frac{\nu}{4}(3+\cos \pi y) \mathbf{I}$ with $\nu>0$. The initial condition is a Gaussian pulse with $R=1 / 7$ and we choose $s$ such that the exact solution of (4.3) reads $u(\mathbf{x}, t)=\frac{1}{4} \exp \left(\left(\mathbf{x}-\mathbf{x}_{0}(t)\right)^{2} / R^{2}-\right.$ $4 \pi \nu t)$ with $\mathbf{x}_{0}(t)=(1 / 2+c t, 1 / 2)^{\top}$.

For the space discretization, we use partitions of the domain built from uniform grids of $m$-by- $m$ squares split into a total of $N=2 m^{2}$ isosceles triangles. Table 4.4 presents the speedup values obtained when varying the polynomial degree, the Peclet number value, and the number of elements. Some results are qualitatively similar to those obtained with the 1D problem in $\S 4.1$. The simplified method keeps the accuracy of the numerical scheme and the efficiency of the SIMP method increases with $p$. Likewise, the speedup is seen to be almost independent of the mesh size and to slightly decrease with $R e_{h}$. However, we note that the speedup values are larger than 


\begin{tabular}{|c|l|c|l|c|c|c|c|}
\hline & & & & & & \multicolumn{2}{|c|}{$\left\|u-u_{h}\right\|_{2}$} \\
\hline$p$ & $N$ & $p_{s}$ & $R e_{h}$ & $\sigma$ & speedup & FULL & SIMP \\
\hline 1 & 3200 & 0 & 100 & 0.2 & 1.61 & $1.80352 E-04$ & $1.80351 E-04$ \\
2 & 3200 & 0 & 100 & 0.2 & 2.46 & $9.91511 E-06$ & $9.92886 E-06$ \\
3 & 3200 & 0 & 100 & 0.2 & 3.32 & $7.57723 E-06$ & $7.57993 E-06$ \\
4 & 3200 & 0 & 100 & 0.2 & 4.06 & $7.52525 E-06$ & $7.53292 E-06$ \\
5 & 3200 & 1 & 100 & 0.2 & 7.10 & $7.22027 E-06$ & $7.32333 E-06$ \\
\hline 3 & 3200 & 0 & 10 & 0.2 & 6.16 & $5.83359 E-05$ & $5.97768 E-05$ \\
3 & 3200 & 0 & 100 & 0.2 & 3.32 & $7.57723 E-06$ & $7.57993 E-06$ \\
3 & 3200 & 0 & 1000 & 0.2 & 2.53 & $5.16744 E-06$ & $5.16745 E-06$ \\
3 & 3200 & 0 & 10000 & 0.2 & 2.23 & $5.28404 E-06$ & $5.28404 E-06$ \\
\hline 3 & 200 & 0 & 100 & 0.2 & 3.38 & $8.70333 E-05$ & $8.71792 E-05$ \\
3 & 800 & 0 & 100 & 0.2 & 3.43 & $1.97619 E-05$ & $1.97785 E-05$ \\
3 & 1600 & 0 & 100 & 0.2 & 3.41 & $1.09399 E-05$ & $1.09451 E-05$ \\
3 & 3200 & 0 & 100 & 0.2 & 3.32 & $7.57723 E-06$ & $7.57993 E-06$ \\
3 & 5000 & 0 & 100 & 0.2 & 3.38 & $5.82650 E-06$ & $5.82831 E-06$ \\
\hline
\end{tabular}

TABLE 4.4. 2D problem: speedups and error levels observed from experiments with $T=0.25$.

those obtained in the 1D problem and allow significant CPU time savings. Indeed, the computational cost of the SIMP method has been shown to be less expensive than the FULL method by a theoretical asymptotic factor $\left(N_{p} / N_{p_{s}}\right)^{2}$ with $N_{p_{s}}=\Pi_{i=1}^{d}\left(p_{s}+i\right) / i$ for large $N[12]$.

\section{Concluding remarks}

An efficient and robust time integration procedure has been successfully applied to linear unsteady convection-diffusion equations. We have used an explicit-implicit time discretization in which the convective term is treated explicitly and the diffusive term implicitly. Integration in time is achieved by applying a Strang splitting method. For the implicit integration we use an iterative algorithm with the simplified method introduced in [12] and extend it to convection dominated flows. The original method is indeed limited by stability constraints (1.1) for $p=1$. The use of a splitting in time of integrations of convective and diffusive terms allows to remove the second condition in (1.1). When using a second-order accurate integration in time, the first condition in (1.1) is seen to be converted into a constraint on the CFL similar to what was obtained in the limit of pure convection in [6]. A von Neumann analysis shows that the simplification does not alter the stability properties of the numerical scheme for polynomial degrees $p \leq 2$ and numerical experiments support this analysis for larger $p$ values. In terms of CPU time, numerical experiments on $1 \mathrm{D}$ and $2 \mathrm{D}$ problems demonstrate an acceleration of convergence of the numerical solution over a wide range of polynomial degree, mesh size, and Peclet number. The method becomes faster for large number of elements and high polynomial degree. Moreover, it allows significant speedups for $2 \mathrm{D}$ problems, thereby confirming that the method is well suited to large-scale problems.

\section{Appendix A. Amplification matrices of FULL and SIMP methods.}

The vector of DOFs in an element $\kappa$ is assumed to be a Fourier mode $\mathbf{U}_{\kappa}^{(n)}=$ $\boldsymbol{\Xi}^{(n)} e^{i j \theta}$ with $\theta \in(-\pi, \pi]$ and $1 \leq j \leq N$ an integer indicating the position of the element in the domain $\Omega_{h}=\cup_{j=1}^{N}[(j-1) h, j h]$ and $h=1 / N$. The amplification matrix 
is then defined by $\mathbf{\Xi}^{(n+1)}=\mathbf{G} \boldsymbol{\Xi}^{(n)}$, where $\mathbf{G}=\mathbf{G}_{c} \mathbf{G}_{v} \mathbf{G}_{c}$ with $\mathbf{G}_{c}=\mathbf{I}-\mathbf{N}_{c}+\frac{1}{2} \mathbf{N}_{c}^{2}$ and $\mathbf{N}_{c}$ the amplification factor for a first-order forward Euler discretization in time of the convective terms on half a time step. For instance, for $p=1$ we have

$$
\mathbf{N}_{c}=\frac{\sigma}{2}\left(\begin{array}{cc}
1-e^{-i \theta} & 1-e^{-i \theta} \\
-3\left(1-e^{-i \theta}\right) & 3\left(1+e^{-i \theta}\right)
\end{array}\right) \text {. }
$$

The amplification factor for the time discretization of viscous terms depends on the choice of the FULL or SIMP method: $\mathbf{G}_{v}=\mathbf{I}-\left(\mathbf{I}+\frac{1}{2} \mathbf{N}_{v}^{\star}\right)^{-1} \mathbf{N}_{v}$, where $\mathbf{N}_{v}$ corresponds to the amplification factor for a first-order forward Euler discretization in time of the diffusive terms on a time step. For instance, for $p=1$ and the FULL method we have

$$
\mathbf{N}_{v}=\frac{\sigma}{R e_{h}}\left(\begin{array}{cc}
-2 e^{-i \theta}\left(e^{i \theta}-1\right)^{2} & e^{i \theta}-e^{-i \theta} \\
3\left(e^{-i \theta}-e^{i \theta}\right) & 12
\end{array}\right)
$$

and $\mathbf{N}_{v}^{\star}=\mathbf{N}_{v}$. For a DG(1) discretization and the SIMP0 method, the uncoupling of DOFs induces

$$
\mathbf{N}_{v}^{\star}=\frac{\sigma}{R e_{h}}\left(\begin{array}{cc}
-2 e^{-i \theta}\left(e^{i \theta}-1\right)^{2} & 0 \\
3\left(e^{-i \theta}-e^{i \theta}\right) & 12
\end{array}\right)
$$

We refer to [12] for further details on the derivation of $\mathbf{N}_{v}^{\star}$ for the SIMP method for higher values of $p$.

\section{REFERENCES}

[1] J.B. Apoung Kamga and B. Després, CFL condition and boundary conditions for DGM approximation of convection-diffusion, SIAM J. Numer. Anal., 44, 2245-2269, 2006.

[2] D.N. Arnold, F. Brezzi, B. Cockburn, and L.D. Marini, Unified analysis of discontinuous Galerkin methods for elliptic problems, SIAM J. Numer. Anal., 39, 1749-1779, 2002.

[3] F. Bassi, S. Rebay, G. Mariotti, S. Pedinotti, and M. Savini, A high-order accurate discontinuous finite element method for inviscid and viscous turbomachinery flows, in Proceedings of the 2nd European Conference on Turbomachinery Fluid Dynamics and Thermodynamics, R. Decuypere, G. Dibelius (eds.), Antwerpen, Belgium, 5-7, 1997.

[4] G. Chavent and B. Cockburn, The local projection $P^{0} P^{1}$-discontinuous Galerkin finite element method for scalar conservative laws, M2AN Math. Model. Anal. Numer., 23, 565-592, 1989.

[5] B. Cockburn and C.W. Shu, TVB Runge-Kutta local projection discontinuous Galerkin finite element method for scalar conservation laws II: General framework, Math. Comput., 52, 411-435, 1989.

[6] B. Cockburn and C. W. Shu, The Runge-Kutta local projection $P^{1}$-discontinuous Galerkin method for scalar conservation laws, M2AN Math. Model. Anal. Numer., 25, 337-361, 1991.

[7] B. Cockburn and C.W. Shu, Runge-Kutta discontinuous Galerkin methods for convectiondominated problems, J. Sci. Comput., 16, 173-261, 2001.

[8] M. Dubiner, Spectral methods on triangles and other domains, SIAM J. Sci. Comput., 6, 345390, 1991.

[9] N. Kroll, H. Bieler, H. Deconinck, V. Couaillier, H. van der Ven, and K. Sorensen, ADIGMA A European Initiative on the Development of Adaptive Higher-order Variational Methods for Aerospace Applications, Notes on Numerical Fluid Mechanics and Multidisciplinary Design, Springer Verlag, 113, 2010.

[10] P. Lesaint and P.A. Raviart, On a finite element method for solving the neutron transport equation, in Mathematical Aspects of Finite Elements in Partial Differential Equations, de Boor (ed.), Academic Press, New York, 89-123, 1974.

[11] W.H. Reed and T.R. Hill, Triangular mesh methods for the neutron transport equation, Technical Report LA-UR-73-479, Los Alamos Scientific Laboratory, NM, 1973. 


\section{IMPLICIT-EXPLICIT DG METHOD FOR CONVECTION DOMINATED FLOWS}

[12] F. Renac, C. Marmignon, and F. Coquel, Time implicit high-order discontinuous Galerkin method with reduced evaluation cost, SIAM J. Sci. Comput., 34, A370-A394, 2012.

[13] C.W. Shu and S. Osher, Efficient implementation of essentially non-oscillatory shock-capturing schemes, J. Comput. Phys., 77, 439-471, 1988.

[14] G. Strang, On the construction and comparison of difference schemes, SIAM J. Numer. Anal., 5, 506-517, 1968. 